\title{
Le Christianisme Céleste en France et en Belgique
}

\section{Christine Henry et Joël Noret}

\section{(2) OpenEdition}

\section{Journals}

Édition électronique

URL : http://journals.openedition.org/assr/16703

DOI : $10.4000 /$ assr. 16703

ISSN : $1777-5825$

Éditeur

Éditions de l'EHESS

Édition imprimée

Date de publication : 1 septembre 2008

Pagination : 91-109

ISBN : 978-2-7132-2191-0

ISSN : 0335-5985

Référence électronique

Christine Henry et Joël Noret, " Le Christianisme Céleste en France et en Belgique », Archives de sciences sociales des religions [En ligne], 143 | juillet-septembre 2008, mis en ligne le 01 juillet 2011 , consulté le 19 avril 2019. URL : http://journals.openedition.org/assr/16703 ; DOI : 10.4000/assr.16703 


\section{Christine Henry, Joël Noret}

\section{Le Christianisme Céleste en France et en Belgique}

Signe de la visibilité de plus en plus grande des communautés «chrétiennes d'outre-mer » en Europe, le CREDIC ${ }^{1}$ leur consacrait en 1998 son colloque annuel en se demandant si ces Églises étaient à considérer comme « de nouveaux réseaux missionnaires ou des refuges identitaires ". À cette question, l'une des participantes, G. Ter Haar, répondait sans ambages : " personnellement, à partir de mes recherches chez les chrétiens africains aux Pays-Bas et dans une moindre mesure ailleurs en Europe, c'est sans aucun doute la première hypothèse qui est vraie (...) la question de l'identité n'est pas tellement le problème des chrétiens africains en Europe : c'est une question bien plutôt pour les chrétiens indigènes en Europe dont l'identité est apparemment menacée par l'arrivée des chrétiens noirs venant d'Afrique» (2000: 51). Tous les participants à cette rencontre n'affichaient pas une opinion aussi tranchée, mais les différences exprimées tenaient plus à des nuances dans les conceptions de "l'identité » qu'à un doute sur « l'audace évangélique » de ces chrétiens venus d'ailleurs (Gerloff, 2000 : 209). Une des idées centrales qui se dégagent de ce colloque est que la plupart des cultures chrétiennes non européennes se ressentent "comme supérieures, pour ainsi dire par élection divine, à la civilisation européenne décadente et informe » et que leurs représentants " n'aspirent pas nécessairement à s'assimiler à ce genre de civilisation " (Spindler, $2000: 23-24)$. C'est dans le prolongement de ces questions et de ces problématiques que nous nous interrogerons ici sur les formes d'organisation et les logiques d'implantation des paroisses françaises et belges du Christianisme Céleste, une Église que nos parcours d'anthropologues nous ont fait rencontrer d'abord en Afrique et plus particulièrement au Bénin ${ }^{2}$.

1. Centre de recherches et d'échanges sur la diffusion et l'inculturation du christianisme, Lyon.

2. Les données sur le Christianisme Céleste en France proviennent d'enquêtes menées par Christine Henry, celles sur la Belgique d'un travail de terrain réalisé par Joël Noret, de façon discontinue, entre le printemps 2000 et le printemps 2004, en particulier dans l'une des paroisses «bruxelloises » de l'Église. Enfin, nous tenons à remercier Maïté Maskens pour l'actualisation des données sur la Belgique qu'elle a réalisée en novembre 2006. 


\section{De l'Afrique à l'Europe}

L'Église du Christianisme Céleste a été fondée en 1947 à Porto-Novo (Dahomey), par un modeste charpentier, Samuel Bileou Joseph Oshoffa, qui avait fréquenté auparavant l'Ordre sacré des Chérubins et des Séraphins, une des plus anciennes Églises du mouvement aladura ${ }^{3}$, une forme de christianisme prophétique née dans la région de Lagos au début du siècle dernier. Oshoffa fut renvoyé de cette Église et quelques années plus tard, à la suite des visions qu'il reçut, fonda sa propre dénomination dont la doctrine, la liturgie et l'organisation sont fortement inspirées de celles des Chérubins et Séraphins ${ }^{4}$. Les Églises aladura ont repris, à l'origine, l'intégralité de la doctrine chrétienne telle que les Anglicans la diffusaient, et s'élevaient avec encore plus de rigueur contre toutes compromissions avec les religions de la coutume. Les Aladura mettent l'accent sur quatre thèmes, que l'on retrouve dans le Christianisme Céleste : la pureté, la prière, les révélations par rêves et visions et la guérison spirituelle. Contrairement aux prophètes aladura qui l'ont précédé, S. B. J. Oshoffa reçut dès ses premières visions l'ordre de créer une Église "chargée de purifier le monde entier ", « dont les membres n'adoreront que Dieu », et constituant « la dernière barque pour le salut ${ }^{5}$.

La doctrine du Christianisme Céleste, qui s'appuie sur un double fondement, la Bible et les révélations reçues par le prophète fondateur ou par les personnes qui en étaient proches au début de l'Église, ne véhicule aucune vision particulière de l'homme noir, contrairement au harrisme et surtout au kimbanguisme, pas plus qu'elle ne s'appuie à l'origine sur une identité ethnique. Oshoffa, qui encourageait l'expansion internationale de sa religion, était le premier à recommander que les cultes soient célébrés dans la langue des fidèles afin que chacun comprenne les prières et puisse y participer.

Dès 1950, des pêcheurs toffins convertis, que leurs activités amenaient fréquemment au Nigeria, établirent la première paroisse céleste à Makoko (dans la banlieue de Lagos). Parallèlement, un ami d'Oshoffa, Nathanaël Yansunnu, fils du méthodiste qui avait éduqué le prophète, est muté en Côte-d'Ivoire, et y établit, également en 1950, une paroisse céleste. L'une des caractéristiques de l’Église, comparée aux autres Églises aladura, est donc qu'elle a fait ses premiers pas simultanément dans ces trois pays. Le groupe de Makoko se développant

3. Le terme yoruba aladura signifie « celui qui prie » ou " le priant ", c'est un terme d'origine arabe dérivé de dura qui signifie "prière individuelle d'intercession ». Comme beaucoup d'autres mots arabes, il est passé dans le vocabulaire yoruba par l'intermédiaire des Songhay (Peel, 2000).

4. Sur l'histoire du mouvement aladura, on peut consulter Peel (1968), et pour l'histoire des Chérubins et Séraphins, Omayajowo (1982).

5. Ces citations sont extraites de Lumière sur le Christianisme Céleste (pp. 15 et 18), brochure écrite par un fidèle béninois en 1972 pour présenter et défendre l’Église que les catholiques classaient parmi les «sectes ». Ce livret contient aussi le règlement intérieur de l’Église. 
rapidement, Oshoffa prit l'habitude de faire de fréquents séjours au Nigeria. En 1976, quand le régime marxiste-léniniste de Kérékou se durcit, Oshoffa quitte le Bénin pour s'y installer définitivement. Mieux secondé qu'au Bénin par des assistants plus instruits, il organise l'Église et la dote, en 1980, d'une constitution qui établit le "siège international » à la paroisse de Ketu (une autre banlieue de Lagos), le "siège du diocèse du Nigeria " à Makoko, et le «siège suprême " à la paroisse-mère de Porto-Novo. À cette époque, le Christianisme Céleste était déjà implanté dans de nombreux pays africains et européens. En 1979, Oshoffa avait accrédité Phillip Ajose en tant que son représentant auprès des " paroisses d'outre-mer » (Europe, États-Unis, Canada). En 1982, il le nomme Supérieur évangéliste et lui rappelle qu'il fait partie de la «haute instance " qui dirige l'Église dans le monde. En fait, alors qu'auparavant, Oshoffa s'efforçait de maintenir l'équilibre entre les branches nigériane et béninoise de l'Église, il devient clair au début des années quatre-vingt que la balance penche maintenant en faveur du Nigeria. Oshoffa lance à Imeko, le village natal de sa mère (dans le Nord-Ouest de l'État d'Ogun, près de la frontière béninoise), la construction d'une " cité céleste » et pose, en 1983, la première pierre d'une immense cathédrale. À partir de ce moment, c'est à Imeko qu'il organise le pèlerinage céleste annuel de Noël (auparavant organisé au Bénin), au cours duquel le pasteur distribue l'onction, sacrement qui fait monter les Célestes sur l'échelle des grades de leur hiérarchie.

La mort soudaine d'Oshoffa dans un accident de voiture, en 1985, ouvre une crise de succession qui n'est pas résolue à ce jour. La tradition orale de l'Église la plus autorisée et la plus communément admise, au Bénin en tout cas, rapporte que le fondateur avait toujours dit que le Saint-Esprit désignerait son successeur qui pourrait être de n'importe quel grade de l'Église. Cette directive est toutefois inscrite sous une formulation différente dans la Constitution nigériane de l'Église comme dans Lumière sur le Christianisme Céleste, la brochure publiée en 1972 à Porto-Novo : il est dit dans ces deux documents que le Pasteur (titre réservé dans l'Église du Christianisme Céleste à la plus haute autorité) sous l'inspiration du Saint-Esprit désignera son successeur. En l'absence d'une telle désignation, et en acceptant que l'ordre hiérarchique vienne combler ce vide, la majorité des Célestes reconnaît comme nouveau chef de l'Église le Nigérian A. A. Bada, second dans la haute hiérarchie et seul tenant du titre de Suprême évangéliste. Les opposants à l'intronisation de Bada donnent des interprétations différentes de la situation. L'une de ces démarches aboutit à la dissidence de la majeure partie de l'Église béninoise qui choisit B. Agbaossi d'abord comme Régent en 1987 puis comme Pasteur en 1996. Le conflit entre les Églises béninoise et nigériane prend rapidement un tour ouvertement ethno-national, la querelle de succession exacerbant les nationalismes religieux sous-jacents. D'autres dissidences, moins importantes, ont lieu au Nigeria et en Côte-d'Ivoire. Bada meurt en septembre 2000, en laissant un "testament " qui nomme Ajose son 
successeur. Celui-ci est intronisé en mars 2001, mais meurt une semaine plus tard. Dans la confusion qui suit, plusieurs dignitaires de l'Église se déclarent Pasteur, si bien que l'Église a aujourd'hui trois Pasteurs principaux, E. Oshoffa (un fils du prophète, choisi comme Pasteur en 2003 par la tendance légaliste de l'Église issue de la "branche Bada »), P. Maforikan (d'origine béninoise, mais issu de l'Église nigériane et à la tête d'un parti surtout nigérian issu, après la mort d'Ajose, de la dissidence de G. Jesse, l'ancien chef de diocèse très populaire du Nigeria) et B. Agbaossi, ainsi que plusieurs "Pasteurs» mineurs qui n'ont qu'une audience réduite et locale ${ }^{6}$.

En Europe, le Christianisme Céleste fait partie de la première génération d' "Églises africaines indépendantes ». C’est dans les années 1967-1969 qu’une première paroisse est ouverte à Londres ${ }^{7}$, à l'initiative d'un groupe d'étudiants nigérians. Londres reste d'ailleurs jusqu'à aujourd'hui le lieu d'implantation le plus important de l'Église en Europe et même, de manière plus générale, hors d'Afrique. L'ampleur des développements du Christianisme Céleste au Nigeria, ainsi que le poids démographique du Nigeria en Afrique occidentale, région où le Christianisme Céleste compte le plus grand nombre de fidèles, ont rapidement fait des Célestes nigérians le contingent le plus important des Célestes immigrés en Europe et aux États-Unis. Les liens historiques entre le Nigeria et la GrandeBretagne font que celle-ci (et Londres en particulier), deviendra le lieu principal de l'émigration nigériane en Europe ${ }^{8}$. À la fin des années soixante également, le premier groupe de prières céleste en Suisse se forme, puis la première paroisse céleste en Allemagne, à Munich, en 1974 (Adogame, 1998 : 150), toujours principalement à l'initiative de groupes d'étudiants célestes nigérians. En Allemagne, l'Église du Christianisme Céleste aurait même été la première Église africaine à s'implanter (Simon, 2001 : 1). Au milieu des années soixante-dix, l'Église s'implante en France où, comme en Grande-Bretagne, le nombre des paroisses augmente au cours des années quatre-vingt, tant par un phénomène de scission, souvent lié à des enjeux de pouvoir, que suite à la croissance du nombre des membres ${ }^{9}$. Enfin, l'expansion européenne du Christianisme Céleste se poursuit dans les années quatre-vingt-dix, si bien que l'Église est aujourd'hui présente dans presque toutes les capitales et dans plusieurs grands ports d'Europe occidentale (Hambourg, Anvers, Amsterdam, Rotterdam).

6. Pour une analyse des luttes de pouvoir et des conflits de légitimité qui agitent l'Église depuis la mort du prophète-pasteur-fondateur, on peut consulter Adogame (2002b), Mary (2005), Henry (sous presse).

7. Voir Adogame (1998: 145; 2001 : 2). Deux autres Églises de la tradition aladura avaient déjà ouvert des paroisses à Londres à ce moment : la Church of the Lord-Aladura en 1964 et les Chérubins et Séraphins en 1965 (Adogame, 2001 : 2).

8. Aux États-Unis, les migrations nigérianes, également importantes, ont été plus dispersées. Le nombre des paroisses célestes y est beaucoup plus élevé (on en compte environ soixantedix) qu'en Grande-Bretagne (où on en dénombre une quarantaine), mais celles-ci sont, dans le second cas, beaucoup plus concentrées sur Londres et sa banlieue.

9. Comme le montrent, dans le cas londonien, les données présentées par Adogame (1998: $145-150)$. 


\section{Les Célestes en France : de l'unité à l'émiettement}

Trois groupes de prières célestes ont, à l'origine, coexisté à Paris, chacun ignorant l'existence des autres. Le premier avait été formé, en 1974, à l'initiative de Pierre Alokpo, un Béninois ${ }^{10}$ de Porto-Novo étudiant en art dramatique, qui, lorsqu'il fut rejoint par ses cousins, commença à célébrer le culte dominical dans le studio de ces derniers, rue Berzelius dans le XVII ${ }^{\mathrm{e}}$ arrondissement. Ils formèrent bientôt un petit groupe d'une douzaine de personnes d'origines différentes (camerounaise, béninoise, togolaise, ivoirienne). Le deuxième groupe était formé de Nigérians qui se réunissait rue Guy-Mocquet, chez Michael Shobowale, En 1978, ce groupe s'installa au domicile d'un autre Nigérian, Ranty Olorunshola, rue du Ruisseau, dans le XVIII ${ }^{\mathrm{e}}$. À la même époque, un groupe d'une quinzaine de Célestes ivoiriens se réunissait autour de Hyacinthe Sarassoro, étudiant en droit venu passer un diplôme en France.

En 1980, Alokpo, apprenant l'existence des Célestes nigérians, se rendit à leur culte dominical avec l'idée de les inviter à se joindre à son groupe, mais constatant que les Nigérians étaient mieux installés car ils disposaient d'une pièce uniquement consacrée au culte, il décida de les rejoindre avec sa petite troupe et contacta également les Ivoiriens qui acceptèrent de se joindre à eux. Ainsi, dans un esprit d'unité assez rare dans l'histoire de l'Église, fut formée la première paroisse de Paris qui regroupa vite une centaine de fidèles. Shobowale en était le responsable, Alokpo était son adjoint, Olorunshola était chargé paroissial. Il fut décidé de toujours célébrer le culte en français, la langue qui semblait la plus fédératrice, et cet usage s'est maintenu jusqu'à aujourd'hui dans toutes les paroisses. Les cantiques furent traduits et des efforts faits pour uniformiser les pratiques liturgiques des uns et des autres. Mais les problèmes ne tardèrent pas à survenir, comme le note Jonas Ahoyo : "déjà à cette époque se posait le problème de la répartition des postes de responsabilité entre les ressortissants des trois pays (Nigeria, Côte-d'Ivoire et le Bénin) » ${ }^{11}$. Mais la question la plus aiguë fut d'abord celle du local. Rue du Ruisseau, avec l'augmentation des effectifs s'accrut aussi le bruit. Les voisins se plaignirent, obligeant, fin 1980, la paroisse à chercher un nouveau lieu de réunion. Après trois déménagements, le groupe trouva enfin, en 1985, à Saint-Denis, un vieux hangar qu'il put louer et remettre en état. La paroisse-mère s'installa durablement en ces lieux. Entre 1981 et 1985, l'absence d'un lieu permanent aggrava les tendances à la dispersion, car, dans ces pérégrinations, et parfois en en prenant prétexte, le groupe commençait à se

10. Dans toute cette partie de l'article, les indications que nous donnons sur la nationalité des personnes ou des communautés dont nous parlons n'indiquent pas leur nationalité légale mais leur pays d'origine.

11. http://www.chez.com/eccjerusalem/histfrance.html, Le Christianisme Céleste en France. Bref aperçu sur l'histoire de l'Église en France, extrait d'un rapport destiné à la "Conférence de réconciliation » (1993). 
disloquer : les problèmes de locaux sont récurrents dans l'histoire de l'Église en France et jouent un rôle non négligeable dans l'exacerbation des conflits.

D'autres logiques de scission apparaissent cependant comme plus fondamentales. Dès les années quatre-vingt, la conjugaison de rivalités et d'enjeux de pouvoir personnel, d'une part, et de tensions entre les diverses composantes ethno-nationales du monde céleste, d'autre part, semble avoir constitué le moteur de différentes scissions. En 1982, à la demande d'une Antillaise mariée à un Togolais, le prophète Oshoffa envoie un assistant évangéliste Pierre Daga, un Béninois, pour «former » les Antillais. Shobowale, qui ne voit pas d'un bon œil l'arrivée de Daga, plus gradé que lui, refuse de l'accueillir, et ce dernier doit se replier à Grigny où il s'occupe à former les nombreux Antillais recrutés dans le voisinage. Mais Daga baptise les nouvelles recrues, ce qui irrite la paroisse-mère qui sanctionne les responsables. Le groupe cesse alors de se rendre le dimanche à la paroisse-mère, et malgré le départ de Daga, prend de l'ampleur. Il devient peu après une véritable paroisse qui s'installe à Corbeil, en 1984, puis à Champigny, en 1986, sous le nom de paroisse Saint-Michel. Dans le même temps, en 1983, un groupe de prière formé à Saint-Ouen, à l'initiative d'une femme ivoirienne en butte à des accusations d'adultère et d'usurpation de grade religieux, devient la paroisse Oshoffa. La même année, Shobowale revient d'un voyage au Nigeria avec le grade d'assistant évangéliste et une lettre du pasteur le nommant responsable de l'Église de France. Souhaitant alors, conformément aux règles édictées par Oshoffa, se consacrer entièrement à sa tâche spirituelle, abandonner son travail et recevoir une indemnité de la paroisse-mère, il se heurte au bureau de la paroisse et, en 1986, entraînant avec lui une grande partie des paroissiens antillais, il se sépare de la paroisse-mère et fonde à Drancy la paroisse Autel de Vie.

Un comité directeur de l'Église de France fait une brève apparition en 1985, mais c'est le pasteur Bada, après la mort d'Oshoffa, qui réorganise celle-ci en 1987. Il nomme le fils aîné du prophète, Emmanuel Oshoffa, chef de l'Église de France. Shobowale est promu président du conseil national, et Olorunshola et Alokpo sont nommés vice-présidents. Habitant Nancy, Emmanuel Oshoffa n'est pas vraiment impliqué dans la gestion quotidienne de l'Église française, que Shobowale dirige effectivement. La figure tutélaire du prophète se profile cependant derrière celle de son fils, et garantit la forme d'unité qui a alors été trouvée. Il est probable que si un autre des dignitaires avait été nommé à ce poste, l'Église française se serait scindée beaucoup plus tôt. L'unité de surface n'empêche pas, d'ailleurs, le processus de segmentation interne de continuer.

En 1988, la paroisse-mère déménage à Aubervilliers, et Olorunshola la quitte pour fonder la paroisse Saint-Paul à Bondy. D'autres paroisses sont créées dans les années qui suivent. En 1993, J. Ahoyo, fondateur de la paroisse de Bethléem (issue de celle de Champigny) écrit dans un rapport destiné à la "Conférence de réconciliation » tenue en août 1993: «Quand nous analysons les causes des différends qui amènent nos frères et sœurs à la création de nouvelles paroisses, 
nous pourrons citer : 1 - la guerre des chefs autour des onctions et des postes de responsabilité ; 2 - la gestion de la trésorerie (cause toujours inavouée); et enfin 3 - l'incapacité de résoudre les problèmes de délits d'adultère. Cette liste n'est malheureusement pas exhaustive ». En effet, après la dissidence de l'Église béninoise, menée par B. Agbaossi, qui suit la mort du prophète en 1985, le bicéphalisme menace l'ensemble des territoires où l'Église est implantée. Cette conférence est organisée pour tenter d'échapper à son extension à l'Église française. Plus tard, dans une interview donnée à La Dernière Barque, un journal céleste béninois, E. Oshoffa déclare : « (...) je ne dépends ni du Bénin, ni du Nigeria. Je suis à la tête de l'Église de France pour défendre son unicité », et il explique : " nos objectifs en initiant une telle Conférence étaient de faire disparaître les désordres qu'occasionnent les visites désordonnées des deux hauts responsables. » En effet, lors de leurs visites, Agbaossi comme Bada distribuaient l'onction, obligeant les fidèles désireux de monter en grade à se déclarer d'un parti ou d'un autre. En 1995, cependant, Bada distribue l'onction en France, puis en 1998 Agbaossi fait de même dans les paroisses qui penchent du côté " béninois " : l'esprit de division a fini par prendre le dessus sur celui de l'unité, Agbaossi dotant même les paroisses qui penchent de son côté d'un nouveau conseil national.

Après le décès du pasteur Bada (2000), les événements qui se sont succédés à la tête de l'Église n'ont guère modifié la donne française, jusqu'au moment de l'intronisation d'E. Oshoffa comme pasteur de la tendance nigériane légaliste de l'Église, en 2003. Le nouveau pasteur, que son parcours prédispose à prendre en compte l'importance des branches non africaines de l'Église, a supprimé le " diocèse d'outre-mer » pour créer autant de diocèses que de pays où l'Église est installée et a nommé, en 2004, Shobowale chef du diocèse de France et des DOM-TOM. En 2005, à la suite de conflits entre ce dernier et d'autres responsables, E. Oshoffa a divisé le diocèse de France en deux zones, Shobowale conservant la direction de l'une d'elles. Parallèlement, Agbaossi reconnut un autre diocèse de France, avec Pierre Alokpo à sa tête. Enfin, la tendance issue de la scission de Jesse (voir supra), qui a aujourd'hui à sa tête le pasteur Maforikan, bien implantée au Royaume-Uni, n'a pas de paroisse reconnue en France.

\section{"Évangéliser les Blancs " : le relais antillais}

L'Église française compte aujourd'hui vingt-cinq paroisses dans la région parisienne, dont la majorité reconnaît l'autorité du pasteur Emmanuel Oshoffa, huit paroisses étant officiellement rattachées à la tendance de B. Agbaossi. Une quinzaine de paroisses sont aussi implantées dans les DOM-TOM (Guyane, Martinique et Guadeloupe). À l'exception de la paroisse Autel de Vie qui a acheté le terrain qu'elle occupe et reconstruit entièrement le bâtiment, les paroisses franciliennes louent très cher des locaux souvent mal adaptés aux fonctions auxquelles ils sont destinés. Par ailleurs, la venue des fidèles occasionnant des allées 
et venues de voitures et les cultes étant nombreux, longs, parfois nocturnes et relativement bruyants, les plaintes du voisinage sont fréquentes et la menace d'avoir à déménager constante. Chacune de ces paroisses compte un nombre restreint de fidèles, un culte dominical ordinaire réunit dix à cinquante fidèles selon la paroisse. D'après leurs propres estimations, les Célestes de France comptent entre mille et mille cinq cents personnes, et sont sans doute aussi nombreux dans les DOM-TOM. Seules les deux plus grandes paroisses peuvent se permettre de rétribuer leur responsable. En dehors de la paroisse-mère qui compte une majorité de Béninois, les fidèles sont d'origines diverses et mélangées. Ils sont en majorité issus des pays d'Afrique sub-saharienne francophones (Côte-d'Ivoire, Togo, Congo, Cameroun) et des Antilles. Les Blancs sont très peu nombreux, et ont généralement connus l'Église par l'intermédiaire d'un conjoint africain ou antillais. Quelques Asiatiques et Maghrébins, presque aussi rares que les Blancs, complètent ce tableau. Au dire des responsables, les fidèles sont mobiles d'une paroisse à une autre et les nouveaux venus difficiles à fixer dans la religion : beaucoup commencent à venir puis abandonnent quelque temps après. Ces comportements laissent à penser qu'en France, comme en Afrique, une logique pragmatique motive les nouveaux fidèles. Recrutés par des relations familiales ou de voisinage qui leur ont laissé entendre que leurs maux s'apaiseraient s'ils fréquentaient l'Église, ils vont voir ailleurs s'ils ne trouvent pas plus rapidement un mieux-être.

Si dans les premiers temps de la formation de la paroisse de Paris, les Célestes immigrés n'avaient pas d'autre but que de se retrouver entre eux pour célébrer leur culte, ils n'avaient pas oublié que leur Église était chargée « de purifier le monde entier ». Dans cette mission, ils ont fait au moins un pas en convertissant des Antillais et en aidant à l'implantation de paroisses dans les DOM-TOM, contribuant par là activement à donner un caractère plus que jamais polycentré à la globalisation du religieux. Les Antillais forment aujourd'hui le contingent le plus important de fidèles dans les paroisses françaises. Certains d'entre eux développent envers leurs " frères en Christ » africains quelques ressentiments car ils estiment qu'ils ne sont pas considérés à leur juste valeur. En témoigne un débat lancé par une Antillaise de la tendance d'E. Oshoffa sur un des sites Internet célestes : la «plaignante » avait appris que Shobowale, suite à sa nomination, préparait un nouvel organigramme du diocèse et avait pressenti deux chefs de district pour diriger l'Église dans les DOM-TOM dont ni l'un ni l'autre n'était antillais : «ce jour, cette communauté se sent ridiculisée, trahie par son Église. L'Antillais n'a-t-il pas la carrure spirituelle pour être chef de district ? Que lui manque-t-il pour être apte à diriger ? " demande t-elle en reconnaissant l'origine africaine de l'Église et des Antillais, mais en arguant aussi de la « culture entièrement différente » de ceux-ci ${ }^{12}$. Cette revendication suscita de nombreuses réponses,

12. "Appel à réaction de tous les chrétiens » (http://fr.msnusers.com/egliseduchristianismeceleste), 13 janvier 2004 . 
presque toutes scandalisées, qui accusaient l'auteur du premier message d'être raciste, de politiser l'Église, de ne pas comprendre la parole christique, et suscita un autre débat qui posait cette question : "à votre avis notre Église est-elle africaine ou universelle ou simplement les deux? "Seule une paroisse francilienne a, à ce jour, été fondée par un Antillais désireux de regrouper sa communauté (et surtout ses membres les plus riches). Mais celui-ci n’a pas été longtemps suivi et les fidèles de cette paroisse, dirigée depuis par un autre Antillais, présentent aujourd'hui la même diversité d'origine qu'ailleurs.

D’une façon plus générale, plusieurs dignitaires africains de l'Église française, qui constatent, comme E. Oshoffa en son temps, que la France « est un pays laïc et très difficile à évangéliser (...) vu le niveau élevé de l'esprit matérialiste ", comptent sur les Antillais pour les relayer. Ils pensent que lorsque ces derniers, dont la culture se situe à mi-chemin de celle des Noirs et des Blancs, seront nombreux à avoir accédé aux postes de responsabilités, ils sauront évangéliser les Blancs ${ }^{13}$. D'autres pensent que les Blancs, habitués à de belles églises, sont rebutés par les conditions d'accueil de la majorité des paroisses célestes et qu'il faut d'abord investir dans l'acquisition de locaux corrects avant de songer à les évangéliser ${ }^{14}$. Quel que soit le niveau de réalisme de ces analyses, elles témoignent du fait que les responsables célestes de l'Église de France considèrent ce pays comme une terre de mission. Ils évoquent d'ailleurs volontiers une prophétie du fondateur selon laquelle c'est à partir de l'Europe que l'Église fera la conquête du monde.

Pour accomplir cette mission, l'Église du Christianisme Céleste souffre d'un déficit de légitimité et de visibilité face à la concurrence des Églises évangéliques et pentecôtistes ${ }^{15}$. D'une part, religion née au Bénin, issue d'une tradition prophétique venue du Nigeria, deux pays ayant eu, jusqu'à une date récente, peu de tradition de migration vers la France, l'Église du Christianisme Céleste est moins « connue » dans ce pays que le harrisme ou le kimbanguisme, et l'opinion commune est plus susceptible d'associer une telle dénomination à la nébuleuse des sectes. D'autre part, le fait que les paroisses célestes sont, en France, fréquentées par des fidèles issus de pays différents les empêche de fonctionner comme des lieux d'entre-soi entre migrants d'une même origine, ce qui limite le recrutement de fidèles sur cette base. La très grande majorité des Célestes français d'origine africaine se sont convertis à cette religion dans leur pays. Enfin, les luttes de pouvoir pour la direction mondiale de l'Église, si elles sont loin d'être spécifiques au Christianisme Céleste, ne servent pas sa cause. On pourrait penser

13. Entretien avec Franck Tutuaku, responsable de la paroisse Grâce Divine (de tendance béninoise) et directeur de publication du journal céleste Le Cep.

14. Entretien avec Jonas Ahoyo, responsable de la paroisse Bethléem, d'un site Internet céleste et de l'association "Secours Céleste".

15. Sur ce point, la situation du Christianisme Céleste en France face à ces Églises est identique à la situation en Belgique, voir infra. 
qu'elles ne concernent guère les fidèles de la base, mais la hiérarchie de l'Église et la manière dont on y progresse, par le biais de l'onction délivrée par le pasteur, font que tout fidèle est obligé de s'en préoccuper, même de façon minimale.

Néanmoins, dans leur travail apostolique, les Célestes français ne sont pas sans atout. Le premier est l'envers du fait que les paroisses n'ont pas de fortes connotations ethno-nationales, elles n'apparaissent donc pas comme des " ghettos " et en sont d'autant plus accueillantes pour différentes populations. Le second est sans doute la stabilité de leurs cadres. Contrairement à la situation en GrandeBretagne et en Allemagne (Adogame, 1998: 145-152, 157), la majorité des Célestes qui ont fondé la première paroisse de Paris est toujours présente à la tête de l'Église française. Pour la plupart venus comme étudiants, ils se sont installés durablement en France même si nombre d'entre eux avouent leur désir de retourner vivre leurs vieux jours en Afrique. Ces dignitaires, qui ont aujourd'hui autour de cinquante ans, font partie de la seconde génération de Célestes, ce sont pour la plupart des enfants des premiers fidèles proches du fondateur. Tout en connaissant bien la tradition de l'Église pour être "nés dedans ", ils ne sont pas obnubilés par sa reproduction à l'identique. Ils affichent des positions d'autant plus réformatrices qu'ils veulent construire l'Église pour leurs enfants qui, probablement, vivront définitivement en France. Pour cette raison, ils sont très soucieux de la reconnaissance de leur Église et désireux de doter le diocèse de France de statuts et d'un financement qui le mettraient à l'abri des remous qui agitent le sommet de l'Église. Il leur faudrait cependant parvenir à surmonter une division que relancent périodiquement les visites pastorales. Cela nous mène à la question de la relation des diocèses périphériques avec les centres pastoraux, un point sur lequel nous reviendrons après avoir considéré la situation de l'Église en Belgique.

\section{L'Église du Christianisme Céleste en Belgique : une marginalité multiple}

En 1991, un musicien béninois, Olivier Jimaja, fonde un groupe de prière céleste à Liège, qui se transforme en une petite paroisse francophone dans la seconde moitié des années quatre-vingt-dix, mais qui ferme en 2001 suite à des problèmes répétés de locaux. Cette première implantation du Christianisme Céleste en Belgique a évolué tout au long de son existence indépendamment d'un second foyer d'implantation, nigérian et anglophone, qui démarre à Bruxelles en 1994. Un contact entre les deux groupes a eu lieu en 1995, mais la fermeture temporaire et un déménagement de la paroisse bruxelloise ont ensuite mené à une perte de contact. En 1996, suite aux enquêtes liées à l'affaire Dutroux ${ }^{16}$, une

16. L'affaire Dutroux, du nom tristement célèbre du meurtrier belge Marc Dutroux, dont la révélation des enlèvements et meurtres d'enfants et de jeunes filles émut les opinions publiques belge et, dans une moindre mesure, européenne, au milieu des années quatre-vingt-dix. 
entreprise d'exploitation de migrants, en particulier nigérians, est découverte, impliquant l'ex-femme de l'un des principaux inculpés de l'époque, Michel Nihoul, et le chargé paroissial de la paroisse céleste bruxelloise d'alors, J. A. Ojegele. Celui-ci, nigérian lui-même, semblait diriger des migrants nigérians cherchant à régulariser leur situation en Belgique vers cette femme, radiée à l'époque du barreau de Bruxelles. Celle-ci, déjà condamnée en 1989 pour des faits d'escroquerie envers des candidats réfugiés politiques, leur demandait alors de fortes sommes d'argent contre la promesse de démarches qui, entamées ou non, n'aboutissaient pas. Le rôle exact du chargé paroissial incriminé (qui n'est plus en Belgique actuellement, mais, à notre connaissance, chargé paroissial au Canada) est aujourd'hui difficile à éclaircir, notamment au regard de la durée de ce circuit. Il semble bien cependant que cet homme percevait à l'époque une commission pour cette "spiritual assistance " particulière et l'orientation des migrants vers ce conseil juridique pour le moins suspect, lequel avait aussi rédigé à l'époque les statuts belges de l'Église, alors enregistrée comme Association Sans But Lucratif (ASBL) sous le nom erroné de "Celestian [au lieu de Celestial] Church of Christ ».

Cette affaire eut à l'époque des répercussions relativement importantes au sein de l'Église du Christianisme Céleste en Europe. Le chargé paroissial fut officiellement désavoué, mais pas exclu. Peu après, lors d'une visite en Angleterre, le pasteur Bada fut brièvement inquiété, l'Église du Christianisme Céleste nigériane, dans son ensemble, ayant pendant un bref moment été suspectée de servir de couverture à un réseau de trafic d'êtres humains ${ }^{17}$. Cet événement, qui précéda de peu la commission d'enquête parlementaire sur les sectes de 1997, contribua aussi probablement à faire inscrire la "Celestian Church of Christ " sur la liste des sectes présentes en Belgique dressée par cette commission (inspirée de l'initiative française de 1995). La promptitude avec laquelle l'Église du Christianisme Céleste a été suspectée de servir de couverture à un réseau pédophile ou/et de traite d'êtres humains (avant d'être rapidement disculpée) est néanmoins significative de l'imaginaire de la «secte » auquel les Églises africaines se trouvent confrontées en Europe ${ }^{18}$.

En 1998, un nouveau chargé paroissial, S. O. Banjo, arrive de Londres, après que la nouvelle avait couru dans les milieux dirigeants de l'Église que la paroisse bruxelloise n'avait plus de responsable. Une révélation a alors enjoint à cet homme de développer l'Église du Christianisme Céleste en Belgique. Résidant

17. Cette affaire n'a pas été la seule dans laquelle des responsables du Christianisme Céleste ont été suspectés ou inculpés de collaboration à des activités de trafic d'êtres humains : on peut citer également le cas d'un chargé paroissial céleste de Benin City (au centre du Nigeria méridional, dans une région où le trafic des mineurs et des femmes est particulièrement concentré) suspecté d'avoir facilité l'envoi de jeunes filles nigérianes en Europe dans le cadre de réseaux de prostitution dans la seconde moitié des années quatre-vingt-dix : voir l'article disponible sur le site www.allafrica.com/stories/200103280132.html (consulté en avril 2003).

18. À propos d'imputations similaires concernant les Églises aladura dans leur ensemble au Royaume-Uni, voir Adogame (2004). 
en Grande-Bretagne depuis le début des années quatre-vingt, Nigérian ayant acquis la nationalité britannique, cadre subalterne dans plusieurs entreprises londoniennes, ce senior evangelist, yoruba comme la grande majorité de l'assistance de la paroisse bruxelloise, entreprend alors un travail de remobilisation du groupe céleste de Bruxelles, dont il cherche à faire croître le nombre. À cette fin, avec une certaine ambition missionnaire qu'il parvient peu à peu à communiquer à ses fidèles, il organise de temps en temps des séances de témoignage pendant le culte, et insiste avec plus ou moins de subtilité sur la nécessité de donner à Dieu pour faire progresser la paroisse et pour être béni en retour ${ }^{19}$. Il est vrai qu'avec une assistance tournant autour d'une vingtaine de personnes chaque dimanche, la paroisse, installée dans d'anciens locaux industriels, éprouve quelques difficultés à se maintenir tout en lui assurant des revenus ${ }^{20}$. En outre, ce nouveau chargé paroissial entre, peu de temps après son arrivée, en conflit puis en procès avec les services sociaux de la commune, qui lui refusent l'accès à toute forme d'aide sociale et font traîner la procédure d'allocation de chômage ${ }^{21}$. Dans cette situation financière difficile, d'autres événements d'ordre privé interviennent dans sa vie, qui le font retourner au Nigeria à l'automne 2002.

C'est pendant les absences de S. O. Banjo, en 2002, que les conflits qui agitent le sommet de l'Église ont les effets en chaîne les plus visibles à Bruxelles. En effet, après le décès inopiné, en mars 2001, du successeur du Pasteur Bada, P. H. Ajose, une semaine seulement après son intronisation officielle, la division affectant l'Église nigériane a créé une certaine instabilité et insécurité institutionnelle, laquelle autorise toutes les tentatives de remise en question des hiérarchies ecclésiales. Pendant cette période, le fondateur du groupe nigérian de l'Église en Belgique (J. A. Ojegele), et, un peu plus tard, un autre des « devanciers » de la paroisse, récemment établi à Bruxelles, sont revenus chacun avec une lettre de Jesse, l'ancien chef de diocèse du Nigeria, les instituant responsables de la paroisse bruxelloise et du diocèse du Benelux. Ces deux tentatives de subversion de la hiérarchie "belge » de l'Église se sont heurtées à l'incompréhension des fidèles réguliers de la paroisse bruxelloise, qui leur ont chaque fois opposé une claire fin de non-recevoir. La seconde tentative a débouché sur la fondation, fin

19. Ne sachant pas où commencer pour toucher un public plus large que la communauté nigériane en Belgique, mais se rendant bien compte de l'obstacle linguistique (les cultes mêlent l'anglais et le yoruba), il propose plusieurs fois à l'un de nous (Joël Noret) de devenir son interprète, et même d'apprendre à conduire les cultes en français.

20. Paul Gifford propose de considérer que le succès de la doctrine pentecôtiste ou néopentecôtiste de la prospérité (qui pousse à donner généreusement à Dieu pour être béni abondamment en retour) doit se comprendre d'abord en raison des avantages qu'elle comporte du point de vue des pasteurs et autres spécialistes religieux eux-mêmes, qui sont évidemment ceux auxquels les dons des fidèles bénéficient directement (2001:65).

21. Âgé d'une cinquantaine d'années et ne parlant aucune des deux langues officielles en région bruxelloise (français et flamand), où le taux de chômage tourne par ailleurs autour de $20 \%$ depuis quelques années, il ne trouve pas de travail. 
2002, d'une deuxième petite paroisse bruxelloise. Quant à la première, elle a fonctionné sans véritable chargé paroissial pendant les années suivantes, jusqu'à ce qu'un nouvel évangéliste en titre prenne le relais en 2006. Mais il n'y avait toujours là, fin 2006, qu'une vingtaine d'adultes qui fréquentaient le culte dominical. Enfin, une troisième paroisse céleste, également dominée par des Nigérians ${ }^{22}$, a vu le jour plus récemment (début 2003) à Anvers (un des ports les plus importants d'Europe), d'où certains membres venaient d'ailleurs auparavant participer au culte du dimanche à Bruxelles. Après quelques mois d'incertitude, cette paroisse a trouvé, fin 2003, la voie d'une stabilité relative, sous la direction de l'ancien secrétaire du pasteur Ajose, K. Awoyemi, alors spécialement dépêché depuis Londres par le chef des diocèses d'outre-mer de l'ex-«branche Bada ", J. O. Oniye (lequel hésitait jusqu'il y a peu entre une fidélité à E. Oshoffa et à P. Maforikan). Cette paroisse anversoise existait toujours fin 2006.

\section{Fluidité et mobilité du monde céleste}

Plus que l'enjeu de la réévangélisation de l'Europe, c'est la question de leur survie qui se pose aux groupes célestes de Belgique. Nous avons noté la méfiance immédiate que le Christianisme Céleste avait rencontrée lorsque son nom apparut suite aux effets en chaîne des enquêtes liées à l'affaire Dutroux, avant que l'affaire ne retombe faute d'indices suffisants. Mais il faut aussi souligner, plus que la méfiance, l'indifférence de la plupart des Belges pour l'offre religieuse d'une Église qui entend renouer avec la lettre du texte biblique, et ce à travers un style liturgique et rituel, relativement spécifique, qui porte largement la marque de son origine africaine. La méfiance envers une institution au nom peu légitime de "Christianisme Céleste » est accentuée par le contexte de discrimination des populations africaines en général. Il y a donc là une première série de facteurs qui ne favorisent pas l'expansion du Christianisme Céleste en Belgique.

À cette première source de marginalisation s'ajoute le fait que l'Église du Christianisme Céleste évolue dans une situation de double minorité dans le champ des Églises africaines en Belgique : la communauté ethno-nationale et linguistique qui la porte autant que le style de christianisme dans lequel elle s'inscrit sont minoritaires. Le Christianisme Céleste est, en Belgique, d'abord attaché aux mondes nigérian et anglophone, alors que dans le pays la grande majorité de la population originaire d'Afrique subsaharienne est francophone et vient d'Afrique centrale (les Congolais d'origine formant assez nettement le

22. Les premiers moments de sa fondation sont cependant liés à la tentative d'un visionnaire béninois de l'Église qui a brièvement cherché à s'installer à Anvers comme visionnaire "professionnel ", cherchant à vivre des services qu'il rendait par l'exercice de son don de vision (on retrouve ici une pratique "professionnelle" de la vision, que le Christianisme Céleste réprouve, mais qui n'est cependant pas exceptionnelle). 
groupe le plus important, voir Maskens et Noret, 2007). Les cultes sont organisés, on l'a dit, en anglais et en yoruba, peu en raison d'une politique identitaire réfléchie ${ }^{23}$, mais bien parce que l'usage de ces langues va en quelque sorte de soi lorsque les fidèles, tous yorubophones et anglophones ${ }^{24}$, se retrouvent entre eux. La barrière linguistique ainsi créée intervient aussi probablement comme facteur supplémentaire de l'absence d'intérêt que le Christianisme Céleste suscite en Belgique auprès des populations non africaines.

Le Christianisme Céleste s'inscrit par ailleurs dans un style de christianisme (celui des Églises aladura) qui, même s'il semble en expansion dans plusieurs parties du continent africain, reste cependant minoritaire. Il est aujourd'hui stigmatisé non seulement par les Églises « historiques ", mais aussi, et peut-être surtout, par la mouvance pentecôtiste qui en diabolise le "syncrétisme ${ }^{25}$. Le recours régulier et abondant dans le Christianisme Céleste à des «objets de puissance » vecteurs de pouvoir mystique (bougies, pagnes, croix ou vêtements bénis) doit ainsi faire face, en Belgique comme ailleurs, aux accusations de "fétichisme » émanant des Églises pentecôtistes qui dominent la scène belge des Églises africaines. Cependant, le fait qu'aucune autre Église aladura ne soit implantée en Belgique confère au Christianisme Céleste une sorte de monopole à ce niveau, qui voit certains fidèles d'Églises proches (par leur style liturgique et leur problématique religieuse, comme celle des Chérubins et Séraphins, ou la Church of the Lord-Aladura) se tourner vers le Christianisme Céleste pendant leur passage, ou lors de leur établissement à Bruxelles.

À cette position minoritaire, à plus d'un titre, du Christianisme Céleste en Belgique s'ajoute encore une double fluidité de l'assistance. Celle-ci provient d'une part des rapports de clientèle ou de fidélisation passagère que le Christianisme Céleste entretient avec une partie de ses fidèles, lesquels ont rejoint l'Église suite à un problème ponctuel, mais peuvent s'en éloigner rapidement si celle-ci ne les satisfait pas. Cette fluidité est générale et se retrouve dans l'ensemble du Christianisme Céleste, mais elle est accentuée en Europe par un second facteur qui est la mobilité relativement importante (favorisée par les accords de Schengen et les politiques de naturalisation rapide de certains pays, qui donnent accès aux avantages des passeports européens) de la population migrante, à laquelle s'adresse principalement le Christianisme Céleste. Ce phénomène a déjà été noté

23. Comme cela semble par contre être le cas dans d'autres Églises africaines, en Europe et sur le terrain africain (Fancello, 2003).

24. Trois fidèles togolais ont un moment fréquenté la principale paroisse bruxelloise en 2002, mais ils ont rejoint la deuxième petite paroisse de Bruxelles lors de sa création, la femme de son chargé paroissial autoproclamé, employée à l'ambassade du Nigeria à Bruxelles, ayant une maîtrise du français très supérieure à celle qu'ont habituellement les Nigérians, qui ne connaissent souvent que quelques phrases de conversation courante.

25. Même si le pentecôtisme fait lui-même, sur le terrain africain, la part belle à des catégories de pensée (sorcellerie, possession, conception persécutrice du mal, etc.) dont l'affinité avec celles des religions "traditionnelles" africaines est souvent troublante (Mary, 2001). 
par B. Simon dans les communautés kimbanguistes et dans les assemblées de la Church of the Lord-Aladura d'Allemagne (2001 : 3). Les données présentées par A. Adogame sur les débuts du Christianisme Céleste en Grande-Bretagne et en Allemagne montrent aussi la mobilité importante des élites ecclésiales et ce dès le commencement de l'implantation européenne de l'Église (1998: 145-152, 157). En Belgique, étant donné la taille restreinte des paroisses célestes, cette double mobilité (religieuse et géographique) des fidèles a pour corollaire l'instabilité des paroisses : seul un très petit noyau de fidèles reste plusieurs années dans une paroisse et peut dès lors être responsabilisé dans sa gestion, ce qui pose rapidement la question du seuil de population à partir duquel une paroisse - même petite et se satisfaisant de locaux minimaux - est viable.

Enfin, la fluidité importante de l'assistance se conjugue également avec la précarité de certaines catégories de migrants, qui sont non seulement mobiles, mais sans ressources. L'assistance aux migrants que peuvent fournir les Églises devient, dans ce contexte, significative. À Bruxelles et à Anvers, les paroisses célestes se voient également dotées d'une dimension supplémentaire : elles deviennent un lieu d'entre-soi privilégié entre migrants d'une même origine, puisque la grande majorité de l'assistance est nigériane et yoruba. Liens religieux, ethno-national et linguistique sont indiscutablement imbriqués. Et les deux derniers peuvent même suffire pour obtenir le secours de la paroisse. En Afrique, les paroisses célestes possèdent des chambres, ou même de petits dortoirs, où peuvent rester, de façon plus ou moins permanente, des fidèles à la situation financière précaire et, de façon ponctuelle, ceux qui ont été invités par la révélation d'un visionnaire, à se mettre en "sécurité ". Dans le prolongement direct de ce type d'organisation, à Bruxelles et à Anvers, des migrants nouvellement arrivés ou dans une situation financière difficile, logent ainsi dans la paroisse. Or, ces « habitants » des paroisses ne sont pas nécessairement célestes. À Bruxelles, au printemps 2004, un seul d'entre eux était céleste, et la moitié était musulmane (et assistait au culte dominical par politesse, sans beaucoup s'y engager), mais tous étaient nigérians et yoruba, comme l'avaient d'ailleurs toujours été les habitants temporaires de cette paroisse bruxelloise. À Anvers, à la même époque, quatre des sept habitants de la paroisse étaient yoruba, dont un céleste, et trois étaient ghanéens, dont un céleste également. On le voit, le lien ethno-national ou la proximité linguistique suffisent pour pouvoir profiter des infrastructures paroissiales et en être reconnu, au moins temporairement, comme un habitant plus ou moins légitime. Les responsables des paroisses célestes, mises à profit par des migrants en situation difficile, justifient d'ailleurs leur accueil par une solidarité "communautaire " non spécifiquement religieuse, fondée surtout sur le partage d'une expérience migratoire dont tous connaissent les aspects pénibles. À Bruxelles, les responsables paroissiaux, qui connaissent des problèmes endémiques de financement de la paroisse, ont aussi essayé de rentabiliser cette présence de migrants en situation difficile dans la paroisse, en leur demandant une 
modique contribution au loyer des locaux, mais sans succès. Et en novembre 2006, les tensions liées aux difficultés de paiement du loyer de la paroisse étaient toujours perceptibles.

\section{Conclusion}

Les exemples contrastés des implantations de l'Église du Christianisme Céleste en France et en Belgique mettent d'abord en évidence les conditions sociales de l'ambition missionnaire. Quand la population susceptible d'être convertie n'est pas suffisamment nombreuse et stable, par exemple, comme c'est le cas en Belgique, les paroisses célestes restent des lieux d'entre-soi de migrants d'une même origine (ou qui se sentent d'origines proches dans le contexte européen). L'ambition missionnaire est dans ce cas limitée, ne concernant pour ainsi dire que les élites ecclésiales, lesquelles y ont aussi des intérêts spécifiques plus évidents. Mais de nombreuses paroisses françaises connaissent également des problèmes endémiques de financement tout en recrutant essentiellement dans des populations "non-Blanches ", conditions d'existence qui contribuent à renvoyer à un horizon très lointain, aux yeux des Célestes comme aux nôtres, un projet missionnaire à destination des sociétés européennes.

L'autre élément sur lequel il nous semble important de revenir est que la création de nouvelles paroisses ne se fait pas sur l'intervention des centres de décision de l'Église. Comme nous l'avons vu, le Christianisme Céleste est une Église fortement hiérarchisée, et idéalement pyramidale. Le pasteur détient, à travers le mécanisme de l'onction (laquelle commande, on l'a dit, les montées en grade dans la hiérarchie ecclésiale), un instrument centralisateur important. Une telle logique institutionnelle n'intervient pas, toutefois, dans la création de nouvelles paroisses, lesquelles surgissent suite à des mésententes ou au terme de parcours migratoires, qui peuvent être relayés par des appels divins reçus par vision. Or, dans la mesure où les instances dirigeantes ne sont pas capables de financer une expansion planifiée, les divers «comités de direction » qui constituent celles-ci sont surtout des lieux d'appropriation de titres honorifiques. Le chargé paroissial de la principale paroisse bruxelloise entre 1998 et 2002 a toujours voulu se faire reconnaître comme "Head of Benelux Diocese ", et ce même à une époque où aucun diocèse du Benelux n'existait ni dans l'un ni dans l'autre des organigrammes des branches nigériane et béninoise de l'Église. Après sa nomination comme Pasteur, E. Oshoffa a confié la direction du diocèse belge à un dignitaire d'origine ivoirienne dont on peut se demander s'il a jamais mis le pied en Belgique. En fait, l'implantation "par le bas » dans de nouveaux territoires est souvent première et remonte ensuite vers le haut de la hiérarchie, lorsque l'affiliation de la (ou des) nouvelle(s) paroisse(s) commence à faire naître des tensions. En effet, les paroisses de Belgique ou de France entretiennent aujourd'hui avec les centres pastoraux une relation paradoxale marquée à la fois 
par des revendications d'indépendance locale (qui prennent facilement appui, en particulier en Belgique, sur l'autofinancement de ces paroisses, lesquelles ne doivent aux centres ni leur création, ni leurs moyens de fonctionnement), et une dépendance ancrée dans le mode de progression dans la hiérarchie ecclésiale et les voies d'accès aux honneurs des grades supérieurs, qui se font via les centres pastoraux. Dans les dernières années toutefois, les revendications d'autonomie locale ont semblé quelque peu prendre le dessus, amplifiées par une certaine disqualification morale des sièges pastoraux qui semblent actuellement faire la preuve de leur incapacité à réunir les membres éclatés de l'Église, dont le prophète fondateur avait pourtant dit, comme on le rappelle souvent, qu'elle avait vocation à demeurer « une et indivisible sous un seul Berger ».

Christine HENRY Paris, CNRS-Centre d'études des mondes africains christ.henry@tiscali.fr

Joël NORET

Bruxelles, Fonds National de la Recherche Scientifique (FNRS)

jnoret@ulb.ac.be

\section{Bibliographie}

Publications de l'Église :

-, 1972, Lumière sur le Christianisme Céleste, Porto-Novo.

-, 1980, Constitution, Nigeria Diocese.

Adogame Afeosemime, 1998, «A home away from home: the proliferation of the Celestial Church of Christ (CCC) in diaspora-Europe ", Exchange. Journal of Missiological and Ecumenical Research, 27-2, pp. 141-160.

-, 2000, "Mission from Africa. The Case of the Celestial Church of Christ in Europe ", Zeitschrift für Missionswissenschaft und Religionswissenschaft, 84-1, pp. 29-44.

-, 2001, "Betwixt Identity and Security: African New Religious Movements (ANRMs) and the Politics of Religious Networking in Europe ", Paper presented at the 2001 International CESNUR Conference in London (http://www.cesnur.org/2001/london2001/adogame.htm), 9 p.

-, 2002a, «Traversing Local-Global Religious Terrain: African Religious Movements in Europe ", Zeitschrift für Religionswissenschaft, 10, pp. 33-49.

-, 2002b, "Legal imbroglios and the post-charismatic fate of the Celestial Church of Christ ", Paper presented at the CESNUR Conference 2002, Salt Lake City and Provo, preliminary version (http:/www.cesnur.org/2002.slc/2002/adogame.htm).

-, 2004, "Engaging the Rhetoric of Spiritual Warfare: the Public Face of Aladura in Diaspora ", Journal of Religion in Africa, 34-4, pp. 493-522.

FANCELLO Sandra, 2003, "Les politiques identitaires d'une Église africaine transnationale. The Church of Pentecost (Ghana) ", Cahiers d'études africaines, 172, pp. 857-881. 
GERLOFF Roswith, 2000, «La diaspora africaine chrétienne en Grande-Bretagne », in Spindler M. et Lenoble-Bart A., (éds.), Chrétiens d'outre-mer en Europe. Un autre visage de l'immigration, Paris, Karthala, pp. 219-228.

GIFFORD Paul, 2001, «The Complex Provenance of Some Elements of African Pentecostal Theology ", in Corten A., Marshall-Fratani R., (éds.), Between Babel and Pentecost: Transnational Pentecostalism in Africa and Latin America, Londres, Hurst \& Company, pp. 62-79.

HENRY Christine, 2008, La force des anges. Rites, hiérarchie et divination dans le Christianisme Céleste (Bénin), Turnhout, BEHE-SR, Brepols.

MARY André, 2001, "Globalisation des pentecôtismes et hybridité du christianisme africain ", in Bastian J.-P., Champion F., Rousselet K., (éds.), La globalisation du religieux, Paris, L'Harmattan, pp. 153-168.

-, 2005, "Histoires d'Église : héros chrétiens et chefs rebelles des nations célestes », in Fourchard L., Mary A., Otayek R. (éds.), Entreprises religieuses transnationales en Afrique de l'Ouest, Ibadan-Paris, IFRA-Karthala, pp. 155-181.

Maskens Maïté, Noret Joël, 2007, « La Nouvelle Jérusalem. Éléments d'histoire et de sociologie d'une Église pentecôtiste en Belgique ", Le figuier. Annales du Centre Interdisciplinaire d'Etude des Religions et de la Lä̈cité, 1, Bruxelles, Éditions de l'Université de Bruxelles, pp. 117-137.

Omayajowo J. Akinyele, 1982, Cherubim and Seraphim. The History of an African Independent Church, Lagos, Nok Publishers International.

PEEL John David Yeadon, 1968, Aladura: A Religious Movement among the Yoruba, London, Oxford, University Press, IAI.

-, 2000, Religious Encounter and the Making of the Yoruba, Bloomington-Indianapolis, Indiana University Press.

Ter HaAr Gerrie, 2000, «Les théories de l'ecclésiogenèse et les diasporas chrétiennes d'outre-mer en Europe ", in Spindler M., Lenoble-Bart A., (éds.), Chrétiens d'outremer en Europe. Un autre visage de l'immigration, Paris, Karthala, pp. 49-66.

SIMON Benjamin, 2001, "Christian Pluralism and the Quest for Identity in African Initiated Churches in Germany ", Paper presented at the 2001 International CESNUR Conference in London (http://www.cesnur.org/2001/london2001/simon.htm), 5 p.

SPINDLER Marc, 2000, « L'implantation d'Église d'outre-mer en Europe : aspects missiologiques ", in Spindler M. et Lenoble-Bart A., (éds.), Chrétiens d'outre-mer en Europe. Un autre visage de l'immigration, Paris, Karthala, pp. 21-34. 


\section{Résumé}

L'Église du Christianisme Céleste (ECC) est aujourd'bui probablement l'Église issue $d u$ mouvement aladura la plus importante dans le monde. Cet article revient sur l'histoire de cette Église prophétique africaine en France et en Belgique, ainsi que sur quelques dynamiques qui traversent les paroisses de l'ECC dans ces deux pays. Ainsi, les situations de l'Église sont à plusieurs égards contrastées. Les paroisses françaises sont plus nombreuses, plus anciennes, et recrutent des fidèles de différentes origines ethno-nationales, tandis que les quelques paroisses belges sont largement fréquentées par des Nigérians. Dans l'un comme dans l'autre cas cependant, les relations que les paroisses entretiennent avec les centres pastoraux de l'Église présentent un caractère paradoxal, et sont marquées à la fois par des revendications d'indépendance locale, et par une dépendance ancrée dans le mode de progression dans la hiérarchie ecclésiale, qui passe par ces centres.

Mots-clés : Église du Christianisme Céleste, France, Belgique, mission, expansion transnationale.

\section{Abstract}

Nowadays, the Celestial Church of Christ (CCC) is probably the most widespread Church of aladura origin. This article offers some details on the history of this African prophetic Church in France and Belgium, and highlights some dynamics that characterize CCC parishes in the two countries. Actually, the respective situations of the Church are quite contrasted. The French parishes are more numerous, more ancient, and recruit faithful of more diverse ethno-national origins, while the few Belgian branches are mostly attended by Nigerians. In both cases however, the relations that the parishes have with the pastoral centres of the Church are somehow paradoxical, and display both claims of local independence, and a dependence grounded in the mode of progression in the ecclesial hierarchy, which depends on these centres.

Key words: Celestial Church of Christ, France, Belgium, mission, transnational expansion.

\section{Resumen}

La Iglesia del Cristianismo Celeste (ICC) es hoy probablemente la iglesia surgida del movimiento aladura más importante del mundo. Este artículo aborda la historia de esta iglesia profética africana en Francia y en Bélgica, así como algunas dinámicas que atraviesan las parroquias de la ICC en Francia y en Bélgica. Así, las situaciones de la Iglesia se comparan en distintos aspectos en los dos países. Las parroquias francesas son más numerosas, más antiguas, y reclutan fieles de diferentes orígenes etno-nacionales, mientras que las parroquias belgas, menos numerosas, son ampliamente frecuentadas por Nigerianos. En un caso como en el otro, sin embargo, las relaciones que las parroquias establecen con los centros pastorales de la Iglesia presentan un carácter paradójico, y están marcadas a la vez por reivindicaciones de independencia local, y por una dependencia anclada en el modo de ascenso en la jerarquía eclesial, que pasa por estos centros.

Palabras clave: Iglesia del Cristianismo celeste, Francia, Bélgica, misión, expansión transnacional. 